\title{
OPEN Altered active control of step width in response to mediolateral leg perturbations while walking
}

\author{
Nicholas K. Reimold ${ }^{1}$, Holly A. Knapp ${ }^{1}$, Rachel E. Henderson ${ }^{1}$, Landi Wilson ${ }^{2}$, \\ Alyssa N. Chesnutt ${ }^{1} \&$ Jesse C. Dean ${ }^{1,2 \bowtie}$
}

During human walking, step width is predicted by mediolateral motion of the pelvis, a relationship that can be attributed to a combination of passive body dynamics and active sensorimotor control. The purpose of the present study was to investigate whether humans modulate the active control of step width in response to a novel mechanical environment. Participants were repeatedly exposed to a force-field that either assisted or perturbed the normal relationship between pelvis motion and step width, separated by washout periods to detect the presence of potential after-effects. As intended, force-field assistance directly strengthened the relationship between pelvis displacement and step width. This relationship remained strengthened with repeated exposure to assistance, and returned to baseline afterward, providing minimal evidence for assistance-driven changes in active control. In contrast, force-field perturbations directly weakened the relationship between pelvis motion and step width. Repeated exposure to perturbations diminished this negative direct effect, and produced larger positive after-effects once the perturbations ceased. These results demonstrate that targeted perturbations can cause humans to adjust the active control that contributes to fluctuations in step width.

In human walking, mediolateral motion of the pelvis during a step predicts step width at the end of the step. This behavior has long been cited as important for ensuring mediolateral walking balance ${ }^{1}$, as a "dynamicallyappropriate step width" that accounts for pelvis motion would presumably be wide enough to prevent a lateral loss of balance toward the stepping leg, but not so wide as to cause excessive mediolateral velocities in the opposite direction during the next step. Indeed, larger mediolateral pelvis displacements and velocities away from the stance foot have been linked to wider steps, as recently reviewed by Bruijn and van Dieen ${ }^{2}$. This relationship can be mathematically quantified using partial derivatives, regressions, or correlations ${ }^{3,4}$, consistently revealing a significant relationship between step width and pelvis motion throughout a step.

However, the existence of statistically significant correlations between pelvis motion and step width is alone not sufficient to prove that the nervous system is actively controlling this relationship, which can be influenced by both active control and the passive dynamics of the body. Passive dynamics (e.g. segment inertial properties) likely play an important role, as simply allowing the stance leg and torso to act as an inverted pendulum could contribute to the observed relationship. Recent 2-dimensional simulations have demonstrated that in the sagittal plane, passive dynamics can produce significant correlations between pelvis motion and step length without need for active control ${ }^{5}$. While similar 3-dimensional simulations require active control to ensure frontal plane gait stability ${ }^{6,7}$, passive dynamics surely contributes to frontal plane motion. This limitation of correlation-based methods has been widely acknowledged ${ }^{2,3,8}$, and has motivated the use of other experimental methods to provide more direct evidence for active control of step width-revealing the existence of clear within-step control. Namely, pelvis dynamics early in a step predicts the magnitude of within-step swing phase hip abductor activity, which in turn predicts mediolateral foot placement location ${ }^{8}$. A similar pattern of active control is seen with the application of mechanical perturbations, as perturbations that increase the pelvis mediolateral displacement or velocity away from the stance leg elicit increased swing phase hip abductor activity and more lateral foot placement ${ }^{8-10}$. Sensory perturbations that create the perception of an altered mechanical state provide further evidence for active control, as perturbations of either hip proprioception ${ }^{11,12}$, vision ${ }^{13}$ or vestibular feedback ${ }^{14}$ are followed by changes in foot placement location consistent with the previously described mechanical principles ${ }^{3}$.

${ }^{1}$ College of Health Professions, Medical University of South Carolina, 77 President St., MSC700, Charleston, SC 29425-571, USA. ${ }^{2}$ Ralph H. Johnson Veterans Affairs Medical Center, Charleston, SC, USA. ${ }^{\circledR}$ email: deaje@ musc.edu 


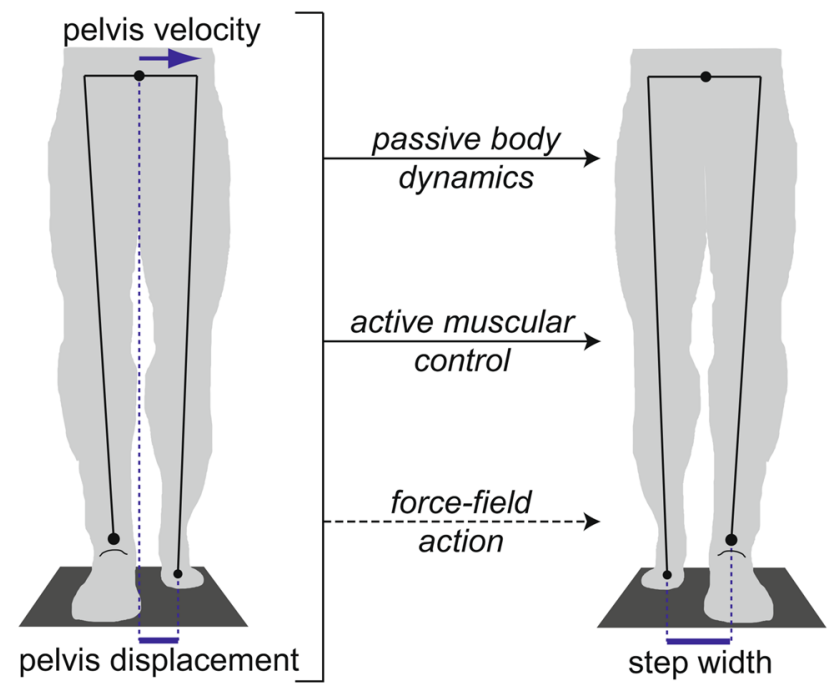

Figure 1. Mediolateral pelvis displacement and velocity at the start of a step (left panel) predict step width at the end of the step (right panel), here illustrated in a frontal plane view of a step with the left foot. This relationship can be influenced by frontal plane passive body dynamics, as the swing leg could act as a pendulum, and the stance leg as an inverted pendulum. Active muscular control can also contribute to this relationship, for instance by using the swing leg hip abductors to influence swing leg position, and the stance leg hip abductors to influence motion of the pelvis relative to the stance leg. In the present study, our force-field is an additional contributor to this relationship, as it can be used to influence step width based on pelvis displacement at the start of the step.

While substantial evidence indicates a role for active control in step width adjustments, it is presently unclear whether humans readily modulate this control to meet the demands of the environment. As a step toward investigating potential changes in active control, we have developed a novel elastic force-field able to push users' legs toward targeted step widths ${ }^{15}$. Walking in this force-field thus involves an additional contributor to the relationship between pelvis motion and step width, as schematically illustrated in Fig. 1. In our initial work, we found that controlling the force-field to produce minimal mediolateral forces on users (Transparent mode) ${ }^{15}$ had a negligible effect on the relationship between pelvis motion and step width ${ }^{16}$. We also used the force-field to "assist" participants toward a dynamically-appropriate step width, based on the motion of their pelvis. This assistive approach had the direct effect of increasing the strength of the relationship between pelvis motion and step width, as quantified using the partial correlation between step width and pelvis displacement at the start of the step (step start $\left.\rho_{\text {disp }}\right)^{16}$. Conversely, we also used the force-field to "perturb" participants, decreasing the strength of the relationship between pelvis motion and step width ${ }^{16}$. Ceasing assistance was followed by short-lived after-effects in which the link between pelvis motion and step width was weakened, while ceasing perturbations caused after-effects in which this link was strengthened ${ }^{16}$.

Extending our prior results, the purpose of the present study was to investigate changes in the previously observed within-step active control of step width upon repeated exposure to an altered mechanical environment produced by our force-field. While the relationship between pelvis motion and step width can be influenced by multiple factors (Fig. 1), our primary comparisons focused on periods in which the force-field action (and passive dynamics of the body) remained constant. Therefore, changes in the relationship between pelvis motion and step width can be attributed to altered active control. Similar approaches of varying mechanical context and quantifying changes in gait kinematics over time have been used extensively to investigate altered control in other novel environments, including split-belt walking ${ }^{17-19}$, walking with leg swing assistance or resistance $\mathrm{e}^{20}$, and walking with leg joint trajectories assisted or perturbed ${ }^{21,22}$. Across these distinct contexts, initial exposure to the novel environment often causes an immediate large change in the movement pattern (here termed a direct effect), which gradually decays back toward baseline as active control is adjusted with repeated experience moving in this environment. Subsequent return to the original mechanical environment often also causes an immediate change in the movement pattern (here termed an after-effect), followed by a return to baseline as active control is again adjusted. With repeated exposure to the same novel environment, the magnitude of both the direct effects and after-effects can decrease, likely as individuals more rapidly adjust their active control to the change in environment ${ }^{23-25}$.

We investigated changes in the relationship between pelvis motion and step width that can be attributed to altered active control, testing four specific hypotheses. First, we hypothesized that upon initial exposure to an altered mechanical environment, the immediate direct effects of the force-field will decrease over time, as participants adjust their active control. Second, we hypothesized that upon the initial removal of the altered mechanical environment, the magnitude of after-effects will decrease toward the baseline level over time. Third, we hypothesized that repeated exposure to the altered mechanical environment will reduce the direct effects produced by the force-field. Finally, we hypothesized that with repeated exposure, the after-effects observed 
(a)

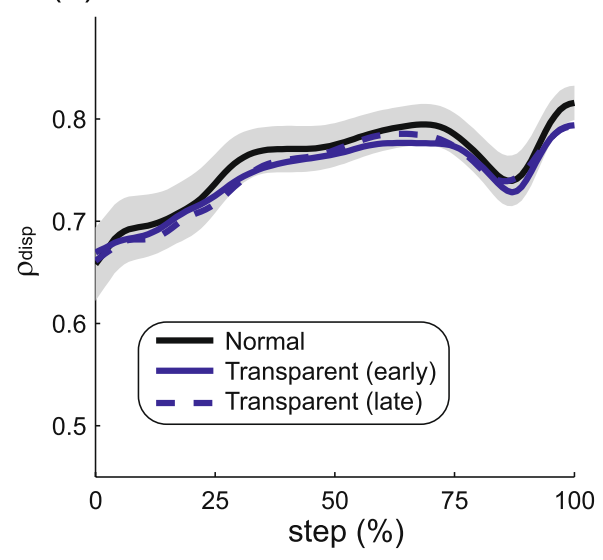

(b)

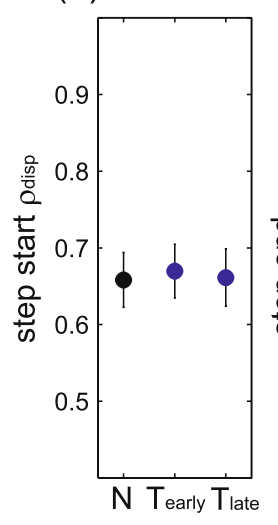

(c)

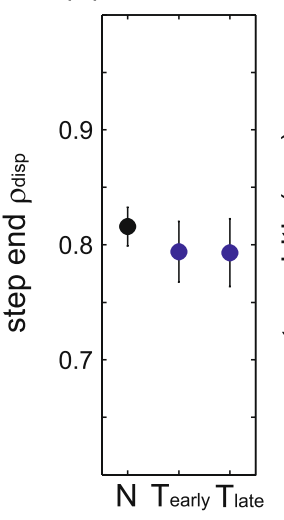

(d)

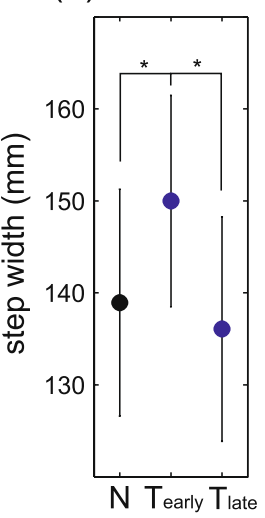

(e)

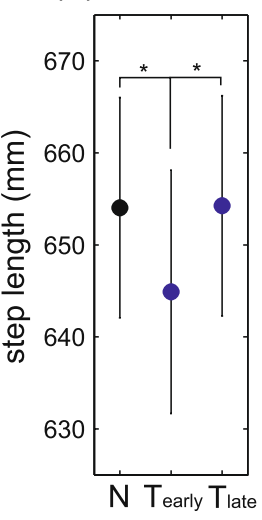

Figure 2. Gait characteristics during initial Normal and Transparent trials. The magnitude of the partial correlation between pelvis displacement and step width $\left(\rho_{\text {disp }}\right)$ is illustrated throughout the step (a) for late in the familiarization Normal trial, early in the Transparent trial, and late in the Transparent trial. Here, "early" refers to the first 45 steps, while "late" refers to steps 181-225. Panels (b-e) illustrate our four gait outcome measures between these Normal $(\mathrm{N})$, early Transparent $\left(\mathrm{T}_{\text {early }}\right)$, and late Transparent $\left(\mathrm{T}_{\text {late }}\right)$ periods. In panel $(\mathbf{a})$, the shaded area indicates the $95 \%$ confidence interval for the Normal period, while confidence intervals are not shown for the Transparent periods to avoid extensive overlap. In panels $(\mathbf{b}-\mathbf{e})$, data points indicate means and error bars indicate $95 \%$ confidence intervals. Asterisks $\left.{ }^{*}\right)$ indicate a significant difference between the indicated periods.

with removal of the force field will decrease. Each of these hypotheses was tested for both force-field assistance and perturbations. As the major focus of this study was changes in the within-step control of step width, our primary measure was step start $\rho_{\text {disp }}$, just as in our prior work ${ }^{16}$. Changes in this correlation would indicate that active control of this within-step relationship has been altered. Secondarily, we quantified the partial correlation between step width and pelvis displacement at the end of the step (step end $\rho_{\text {disp }}$ ). This metric quantifies the extent to which any within-step adjustments influence the relationship between pelvis state and step width once the new base of support is established. The presence of this relationship (e.g. wider steps accompanying larger mediolateral pelvis displacements) is reasonably considered important for ensuring mediolateral walking balance $^{1,2}$, as a too-narrow step could cause a lateral loss of balance. Finally, our secondary analyses also included the more traditional gait metrics of step width and step length.

\section{Results}

Gait behavior prior to force-field assistance or perturbations. While the purpose of this study was to investigate changes in the within-step active control of step width in response to an altered mechanical environment, we first investigated potential changes in gait behavior before assistance or perturbations were applied. Specifically, we compared our gait metrics between the end of a familiarization Normal trial in which participants did not interact with the force field, and early and late in a subsequent 5-min Transparent trial (with minimal mediolateral forces). The relationship between mediolateral pelvis motion and step width did not vary substantially across these walking periods (Fig. 2a). Neither step start $\rho_{\text {disp }}\left(p=0.94 ;\right.$ Fig. 2 b) nor step end $\rho_{\text {disp }}$ ( $p=0.94$; Fig. $2 c$ ) were significantly different across these three periods. In contrast, step width varied significantly ( $p=0.002$; Fig. $2 \mathrm{~d}$ ), as participants walked with wider steps early in the Transparent trial, but returned to the baseline Normal value by late in this trial. Similarly, step length varied significantly $(p<0.001$; Fig. 2 e), with shorter steps early in the Transparent trial that returned to the baseline Normal value by late in this trial. The lack of a change in $\rho_{\text {disp }}$ indicates that interfacing with the force-field in Transparent mode did not cause a change in the active control of step width, despite clear changes in mean step width and length.

Gait changes spanning three force-field exposures. For illustrative purposes, we here depict the changes in each gait metric across three exposures to force-field assistance or perturbations, interspersed with washout periods in Transparent mode. The clearest effects on step start $\rho_{\text {disp }}$ (Fig. 3a) and step end $\rho_{\text {disp }}$ (Fig. 3b) were observed during the periods in which assistance or perturbations were applied, with assistance increasing and perturbations decreasing $\rho_{\text {disp }}$. During the washout periods, the $\rho_{\text {disp }}$ metrics returned toward their baseline level (dashed line), sometimes exhibiting overshoot beyond this value. In general, step width tended to decrease over time (Fig. 3c), while step length increased (Fig. 3d). The increases in step width and decreases in step length relative to baseline were more apparent during the periods with assistance than those with perturbations.

Direct effects during the first force-field exposure. Force-field assistance increased the strength of the relationship between pelvis displacement and step width $\left(\rho_{\text {disp }}\right)$ throughout a step (Fig. 4a). Over the duration of the first exposure to the assistance, step start $\rho_{\text {disp }}$ decreased significantly $(\mathrm{p}=0.015$; Fig. $4 \mathrm{~b})$, while remaining higher than the baseline Transparent level. Conversely, we did not observe a significant change in step 


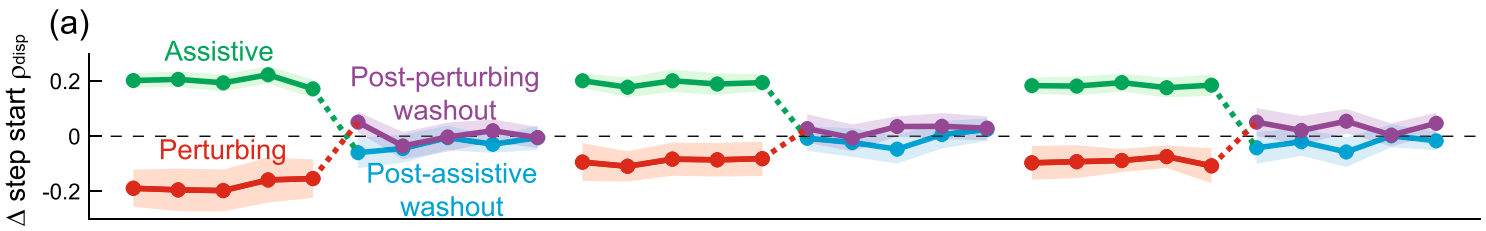

(b)
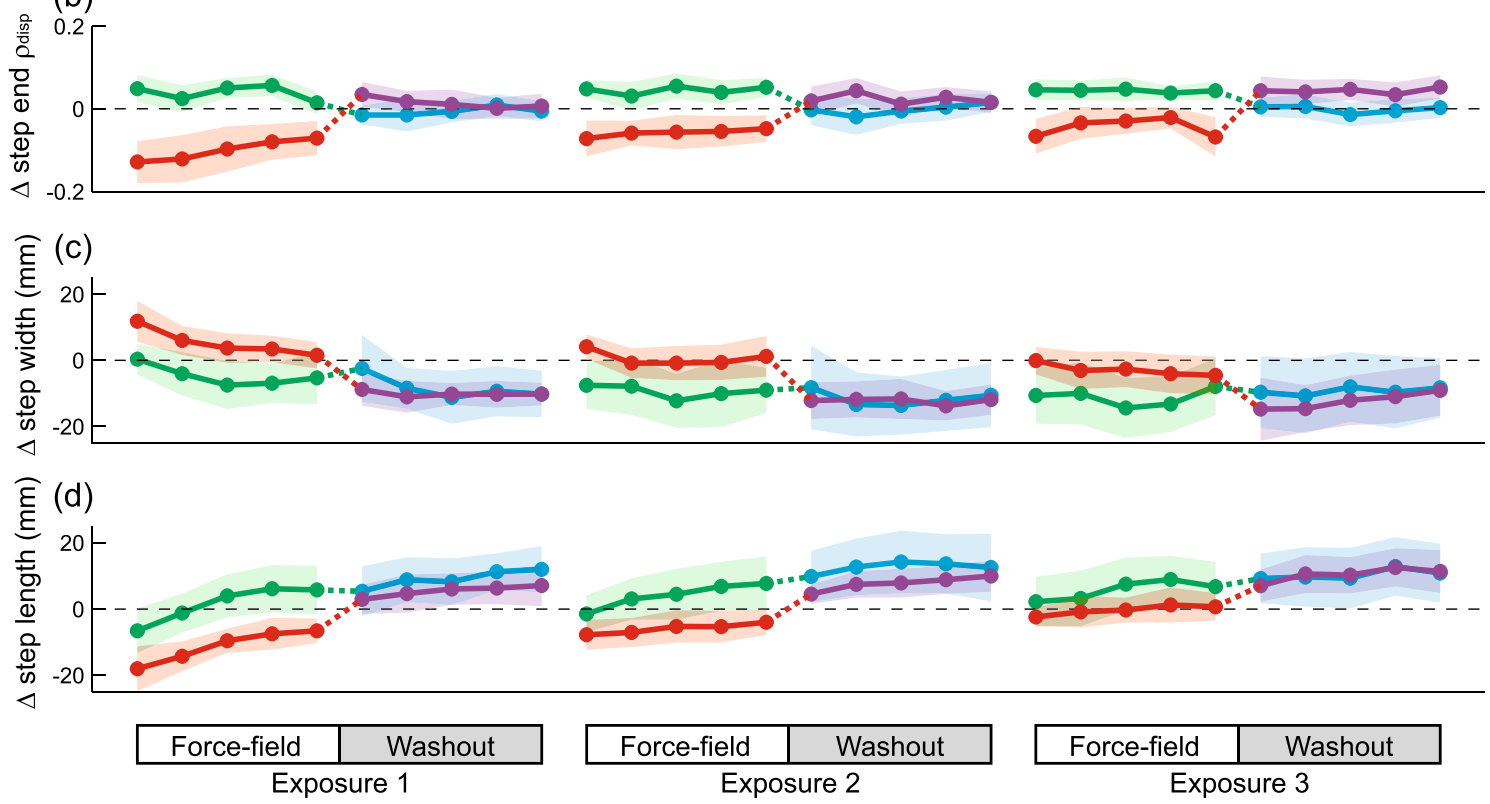

Figure 3. Gait behavior across three consecutive 10-min walking trials (exposures) that included either forcefield assistance or perturbations. These changes are illustrated for step start $\rho_{\text {disp }}$ - the partial correlation between pelvis displacement and step width $(\mathbf{a})$, step end $\rho_{\text {disp }}(\mathbf{b})$, step width (c), and step length $(\mathbf{d})$, and are plotted in terms of the difference from the initial Transparent trial. Each data point represents the mean value calculated within each 45 -step bin, and shaded areas indicate $95 \%$ confidence intervals. The dashed line at zero is presented to allow easier visualization of changes relative to the initial Transparent trial.

end $\rho_{\text {disp }}(p=0.063$; Fig. $4 c)$. Step width did not change ( $p=0.13$; Fig. $\left.4 d\right)$ across the initial exposure to forcefield assistance, while step length increased significantly $(p=0.002$; Fig. 4e). Opposite to the effects observed with force-field assistance, perturbations weakened the relationship between pelvis displacement and step width throughout a step (Fig. 4f). This decrease in step start $\rho_{\text {disp }}$ did not change over the course of this initial trial $(p=0.36$; Fig. $4 \mathrm{~g})$, while step end $\rho_{\text {disp }}$ increased significantly closer to its baseline value $(p=0.018$; Fig. $4 \mathrm{~h})$. Both step width $(p<0.001$; Fig. 4 i $)$ and step length $(p=0.001$; Fig. 4 j) changed significantly over the course of the perturbation trial, with step width decreasing and step length increasing. Participants' gait behavior across their first exposure to force-field assistance or perturbations thus produced equivocal evidence for changes in active control; while the direct effects of both force-field methods on $\rho_{\text {disp }}$ tended to decrease toward baseline over time, these changes did not consistently reach statistical significance.

After-effects during the first washout period. Following the first exposure to force-field assistance, the magnitude of $\rho_{\text {disp }}$ throughout the step did not vary from early to late in the subsequent washout period (Fig. 5a). No significant differences were observed for step start $\rho_{\text {disp }}\left(p=0.14\right.$; Fig. 5b) or step end $\rho_{\text {disp }}(p=0.55$; Fig. 5c) between these time periods. However, step width decreased significantly $(p=0.016$; Fig. $5 \mathrm{~d})$ and step length increased significantly ( $p=0.012$; Fig. $5 \mathrm{e}$ ) over this same period. Over the course of the first washout period after force-field perturbations, $\rho_{\text {disp }}$ magnitude decreased significantly toward its baseline value (Fig. $5 f$ ), as observed for step start $\rho_{\text {disp }}\left(p=0.030\right.$; Fig. 5g) and step end $\rho_{\text {disp }}\left(p=0.040\right.$; Fig. 5h). For both of these metrics, $\rho_{\text {disp }}$ magnitude did not differ from the baseline Transparent trial by late in the washout period. Step width did not change $(p=0.52$; Fig. 5i) from early to late in the first washout trial, while step length increased significantly $(p=0.009$; Fig. $5 j$ ). The observed changes in $\rho_{\text {disp }}$ during this period in which the mechanical context remained constant indicate that the active control of step width was initially altered following perturbation exposure before gradually returning to baseline, while any such changes in active control were not as apparent following assistance.

Direct effects with repeated force-field exposure. Across all three exposures to force-field assistance, $\rho_{\text {disp }}$ magnitude throughout the step remained elevated relative to its baseline value (Fig. 6a). No significant differences were observed across these exposures in terms of direct effects on step start $\rho_{\text {disp }}(p=0.27$; Fig. 6b) or step end $\rho_{\text {disp }}(p=0.96$; Fig. $6 c)$. Step width decreased significantly across these exposures $(p=0.009$; Fig. $6 \mathrm{~d})$, 
(a)

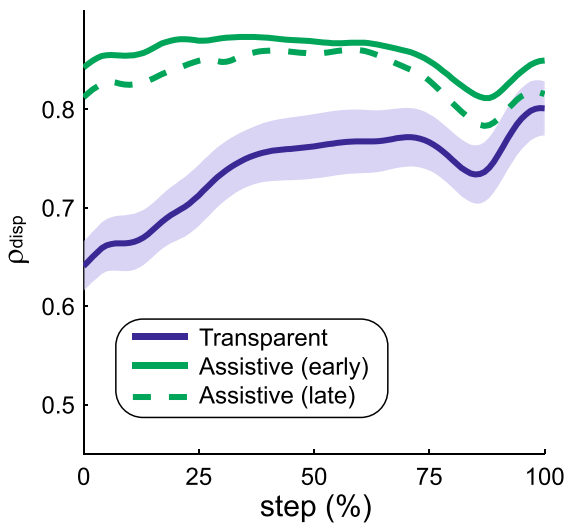

(f)

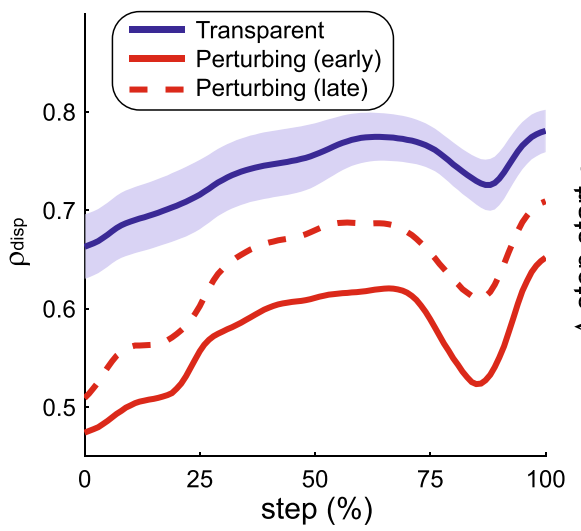

(b)

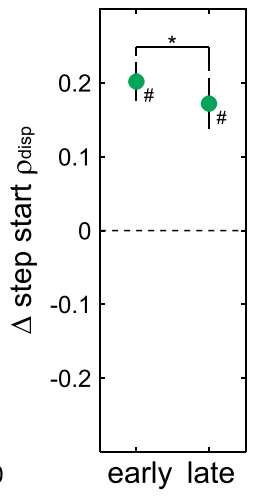

(g)

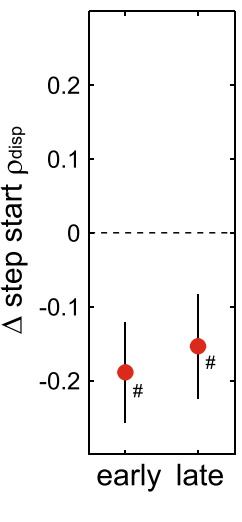

(c)

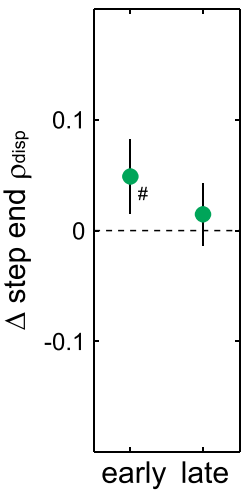

(h)

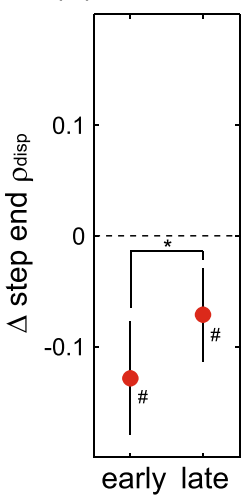

(d)

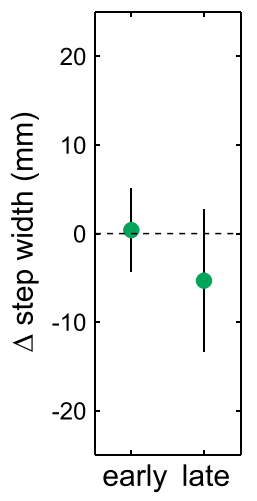

(i)

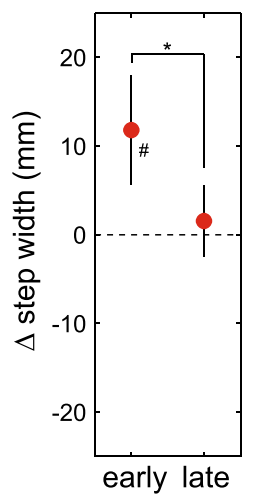

(e)

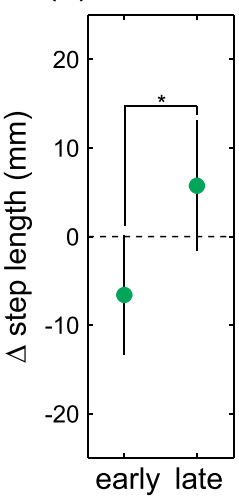

Figure 4. Direct effects of the first force-field exposure. The top row illustrates the direct effects of force-field assistance, in terms of the magnitude of the partial correlation between pelvis displacement and step width $\left(\rho_{\text {disp }}\right)$ throughout the step $(\mathbf{a})$ and our four gait outcome measures $(\mathbf{b}-\mathbf{e})$. The bottom row $(\mathbf{f}-\mathbf{j})$ follows the same structure to illustrate the direct effects of force-field perturbations. For all comparisons, "early" refers to the first 45 steps, while "late" refers to steps 181-225. In panels (a) and (f), the shaded area indicates the 95\% confidence interval for the initial Transparent trial, while confidence intervals are not shown for the experimental conditions to avoid extensive overlap. For the remaining panels, data are presented as the difference from the initial Transparent trial. Data points indicate means and error bars indicate $95 \%$ confidence intervals. Asterisks $\left.{ }^{*}\right)$ indicate a significant difference between the indicated early and late periods. Pound signs (\#) indicate a significant difference from the initial Transparent trial, with the $95 \%$ confidence interval not including zero (dashed line).

while step length did not change ( $p=0.56$; Fig. 6e). Changes in the direct effects of repeated force-field exposure were more apparent with perturbations. The $\rho_{\text {disp }}$ magnitude throughout the step was decreased relative to its baseline value for all exposures but was closer to baseline during the second and third exposures (Fig. 6f). For both step start $\rho_{\text {disp }}(p=0.044$; Fig. $6 \mathrm{~g})$ and step end $\rho_{\text {disp }}(p=0.030$; Fig. $6 \mathrm{~h})$, the direct effects of perturbations were significantly smaller in later exposures. Repeated exposure also significantly influenced the direct effects of perturbations on step width $(p<0.001$; Fig. 6i) and step length $(p<0.001$; Fig. 6j), as step width decreased and step length increased in later exposures. These results indicate that repeated perturbation exposure caused a change in the active control of step width, whereas no such effect was observed with repeated assistance.

After-effects with repeated force-field exposure. The observed $\rho_{\text {disp }}$ magnitudes throughout the step were similar during each of the washout periods following force-field assistance (Fig. 7a). No significant differences were present between these washout periods in terms of step start $\rho_{\text {disp }}\left(p=0.58\right.$; Fig. 7b) or step end $\rho_{\text {disp }}$ $(p=0.75$; Fig. 7c). Similarly, no significant differences were present between the washout periods for step width ( $p=0.26$; Fig. $7 d)$ or step length $(p=0.56$; Fig. 7e). During the washout periods following force-field perturbations, $\rho_{\text {disp }}$ magnitudes throughout the step increased for later exposures (Fig. 7f). Both step start $\rho_{\text {disp }}(p=0.042$; Fig. 7g) and step end $\rho_{\text {disp }}$ increased significantly $(p=0.014$; Fig. 7h) following later perturbation exposures. Step width did not vary across consecutive washout periods ( $p=0.17$; Fig. $7 \mathrm{i})$, but step length increased significantly $(p=0.017$; Fig. $7 \mathrm{j})$ in later washout periods. As with the direct effects of repeated exposure, these results indicate that changes in the active control of step width were sustained beyond the cessation of perturbations, but not following assistance. 
(a)

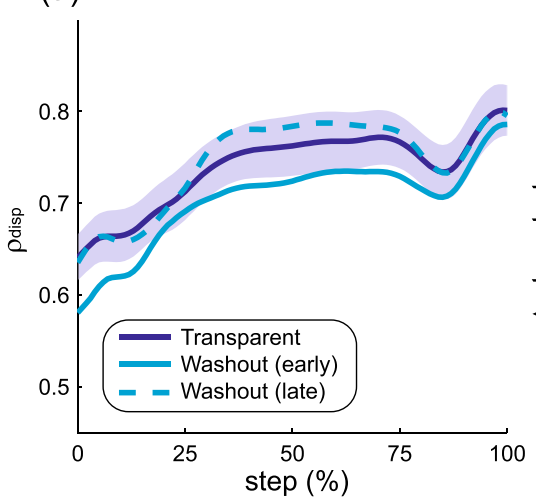

(f)

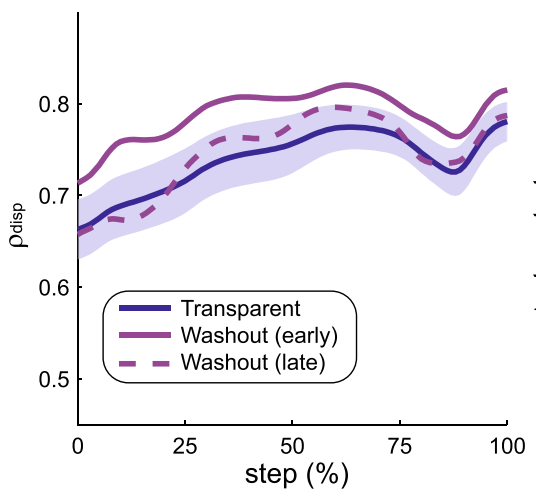

(b)

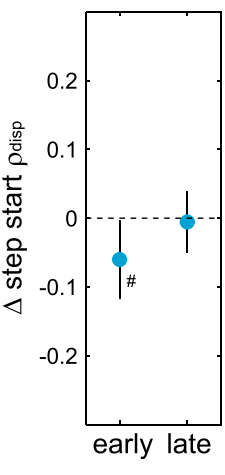

(g)

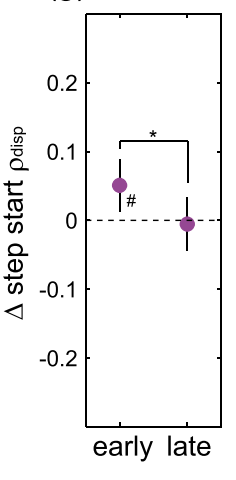

(c)

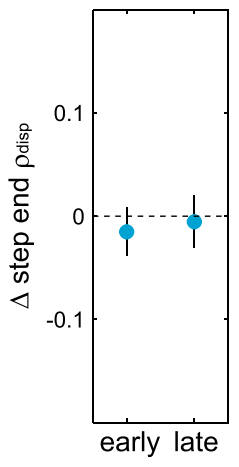

(h)

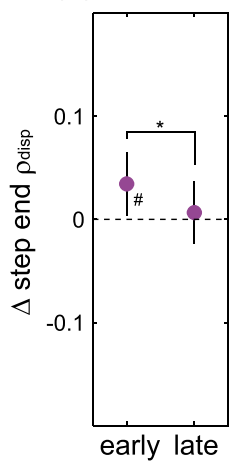

(d)

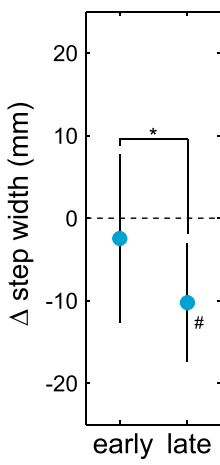

(i)

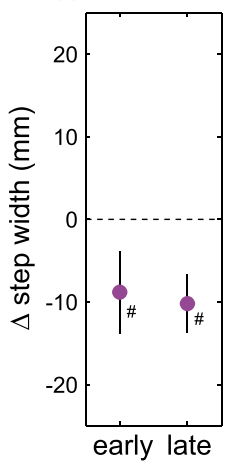

(e)

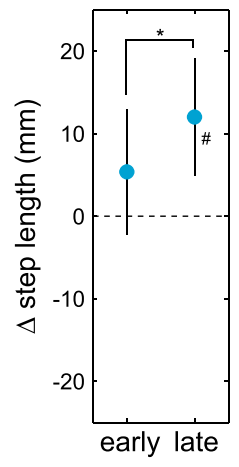

Figure 5. Gait behavior over the course of the first washout period. The top row (a-e) illustrates potential after-effects of force field assistance, while the bottom row $(\mathbf{f}-\mathbf{j})$ illustrates potential after-effects of force-field perturbations. For all comparisons, "early" refers to the first 45 steps, while "late" refers to steps 181-225. The structure of the figure is the same as that described for Fig. 3, with $\rho_{\text {disp }}$ representing the partial correlation between pelvis displacement and step width.

\section{Discussion}

Exposure to a novel force-field caused changes in the relationship between mediolateral pelvis motion and step width. An increase in $\rho_{\text {disp }}$ (our measure of this relationship) indicates a stronger link between these mechanical variables, and a decrease in $\rho_{\text {disp }}$ indicates a weaker link. In the context of the present study, we attribute changes in $\rho_{\text {disp }}$ to changes in the active control of step width, as our comparisons focused on periods in which the force-field action and the passive dynamic properties of the body remained constant. While both force-field assistance and perturbations had measurable effects on $\rho_{\text {disp }}$, our hypotheses were only partially supported. Our first hypothesis that the immediate direct effects of the force-field would decrease toward baseline over the course of the first exposure was partially supported; the direct effects of assistance decreased significantly for step start $\rho_{\text {disp }}$ but not step end $\rho_{\text {disp }}$, while the direct effects of perturbation decreased significantly for step end $\rho_{\text {disp }}$ but not step start $\rho_{\text {disp. }}$. Our second hypothesis that the after-effects of the first force-field exposure would decrease toward baseline upon removal of the novel mechanical environment was supported for perturbations, but not assistance. Our third hypothesis that the direct effects of the force-field would decrease with repeated exposure to the novel mechanical environment was also supported for perturbations, but not assistance. Finally, our fourth hypothesis that the after-effects of the force-field would decrease with repeated exposure was not supported; the after-effects following perturbations actually increased with repeated exposure.

While the present study was not a direct replication of our previous force-field experiments, the changes in gait behavior observed upon initial exposure to assistive or perturbing forces were consistent with our prior results. Specifically, we previously found that force-field assistance (across varied control equations) directly strengthened the link between pelvis motion and step width, as quantified with $\rho_{\text {disp }}$ at the start of the step ${ }^{16}$. Upon cessation of the assistance, we observed short-lived after-effects in which step start $\rho_{\text {disp }}$ was decreased relative to baseline. In contrast, force-field perturbations weakened the link between pelvis motion and step width, and caused subsequent short-lived positive after-effects ${ }^{16}$. The same patterns of direct effects and after-effects on step start $\rho_{\text {disp }}$ were seen in the present study upon the first exposure to assistance or perturbations.

Here, our focus was on changes in gait behavior across extended periods of walking while the force-field remained in the same control mode (i.e. Transparent, Assistive, Perturbing). Our primary metric quantified the relationship between pelvis displacement at the start of the step and step width. While multiple factors can contribute to the strength of this within-step relationship (including passive dynamics) ${ }^{2,3,8}$, comparing periods 
(a)

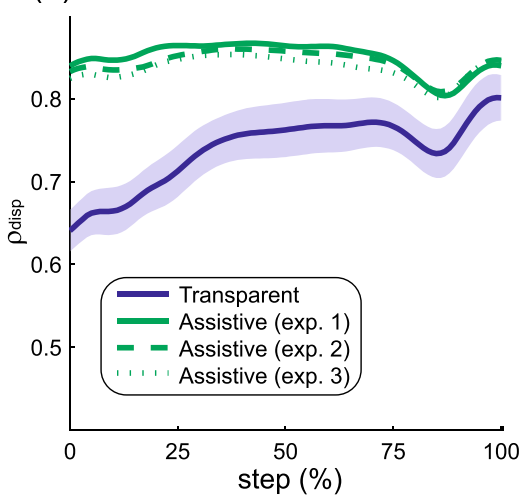

(f)

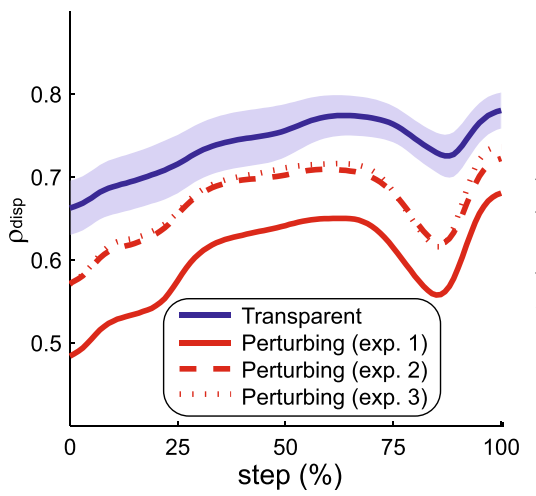

(b)

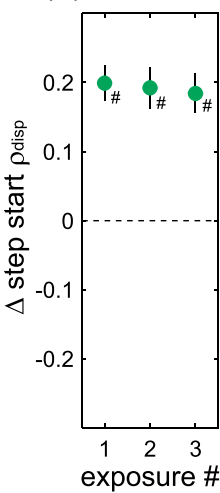

(g)

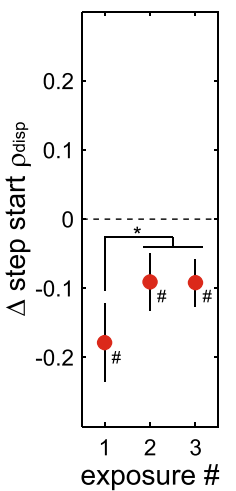

(c)

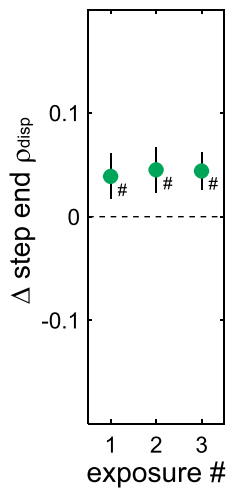

(h)

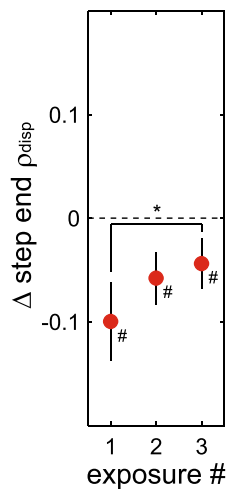

(d)

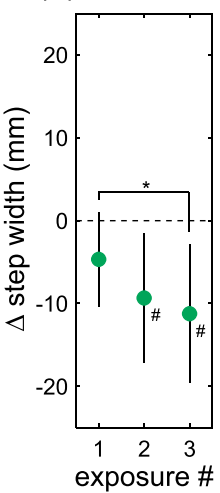

(i)

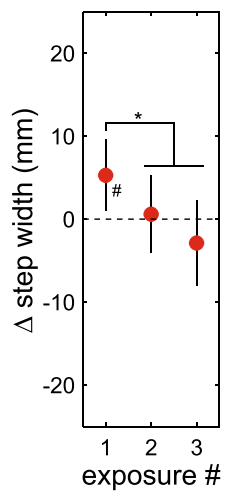

(e)

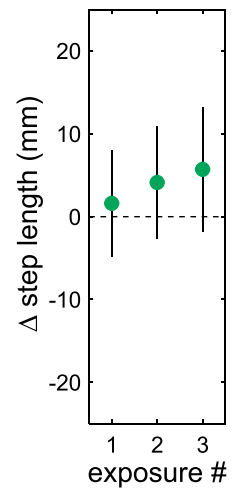

(j)

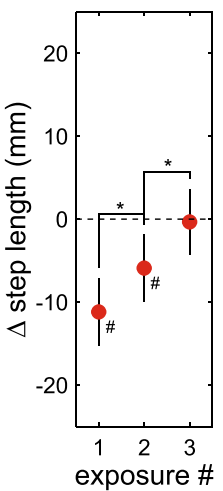

Figure 6. Effects of repeated exposure to the force-field. Following the structure of Fig. 3, the top row (a-e) illustrates the direct effects of force-field assistance, while the bottom row $(\mathbf{f}-\mathbf{j})$ illustrates the direct effects of force-field perturbations. As in previous figures, $\rho_{\text {disp }}$ represents the partial correlation between pelvis displacement and step width. For these comparisons, the first 225 steps of each exposure are included. Asterisks ${ }^{*}$ ) indicate significant differences between the indicated exposures. Pound signs (\#) indicate a significant difference from the initial Transparent trial.

in which the mechanical context remained the same allows us to attribute changes to altered active control. Secondarily, we quantified the relationship between pelvis displacement at the end of the step and step width, providing insight into the ultimate effect of any within-step adjustments. This metric is conceptually similar to variability in the mediolateral margin of stability calculated from the body's extrapolated center of mass ${ }^{3}$, a common measure of walking balance. Importantly, neither of these metrics (step start $\rho_{\text {disp }}$ and step end $\rho_{\text {disp }}$ ) changed from early to late in the initial Transparent trial, indicating that any observed changes are not simply due to altered active control with extended periods of walking.

Exposure to force-field assistance produced only weak evidence for changes in active control, with qualitatively similar effects for step start $\rho_{\text {disp }}$ and step end $\rho_{\text {disp. }}$. Over the course of the first exposure to assistance, $\rho_{\text {disp }}$ tended to decrease toward its baseline value, although this decrease did not reach significance for step end $\rho_{\text {disp. }}$. The reduction in step start $\rho_{\text {disp }}$ may be a result of the assistance allowing participants to safely exert less active control over this within-step relationship - a "slacking" phenomenon previously observed with assistive robotic orthoses $^{26}$. However, the hypothesized changes in $\rho_{\text {disp }}$ during the first washout period and with repeated exposure to assistance were not directly supported by our results. Across all three exposures, $\rho_{\text {disp }}$ remained elevated while assistance was applied, and essentially returned to baseline when the assistance ended. The lack of large changes in $\rho_{\text {disp }}$ across repeated bouts may be due to the assistive nature of the forces, as mechanical contexts that challenge walking balance appear more likely to cause adjustments in active control than contexts not perceived as challenging ${ }^{27}$. The present results also provide no evidence for changes in active control that would cause the altered movement pattern (with increased $\rho_{\text {disp }}$ ) to be retained once the assistance ends, consistent with prior results in which force-fields were used to assist achievement of a specific footpath trajectory during walking ${ }^{21,22}$.

Force-field perturbations were often accompanied by changes in $\rho_{\text {disp }}$ indicative of altered active control. The direct effect of perturbations was to weaken the link between pelvis displacement and step width; however, this relationship tended to return toward its baseline level with extended or repeated periods of exposure. Similar results have been observed with force-fields that perturb the footpath trajectory during walking ${ }^{22,28}$. While we did not observe a significant increase in step start $\rho_{\text {disp }}$ from early to late in the first perturbation exposure, this may have simply been due to the relatively short (5-min) duration of this first exposure. Indeed, changes in gait kinematics during spit-belt walking can continue to develop across five 15-min exposures on consecutive days ${ }^{25}$, 
(a)

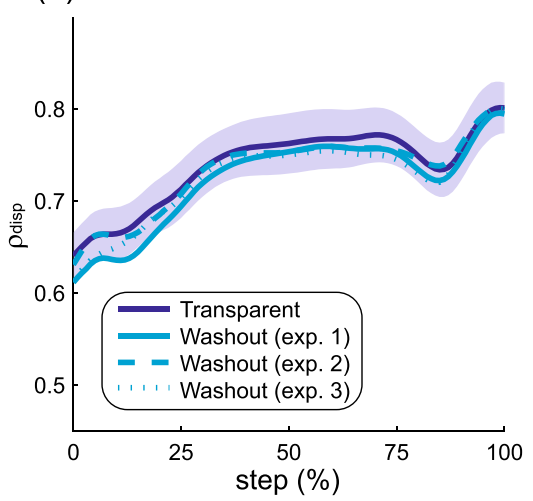

(f)

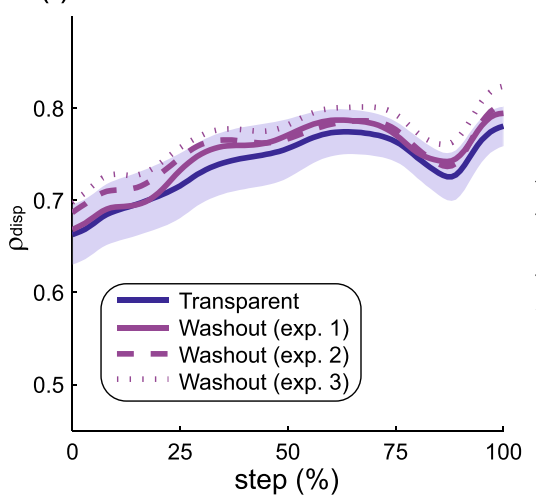

(b)

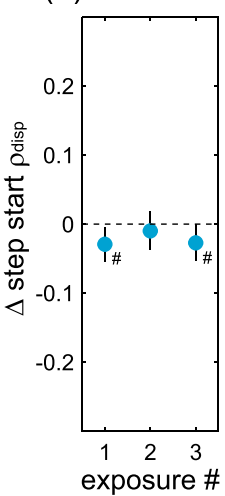

(g)

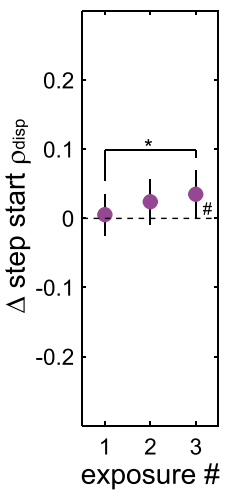

(c)

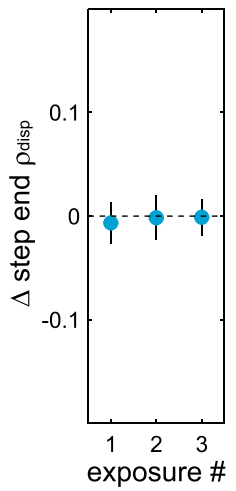

(h)

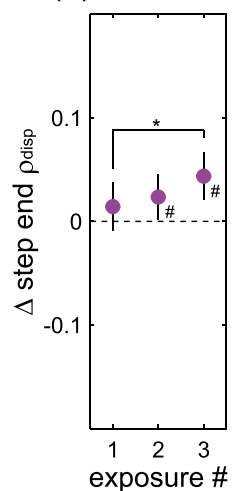

(d)

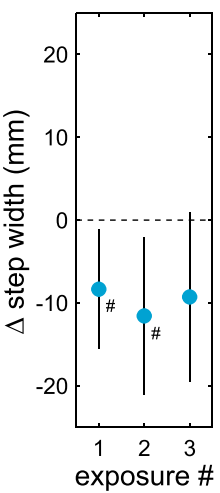

(i)

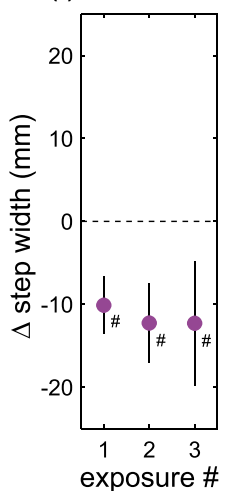

(e)

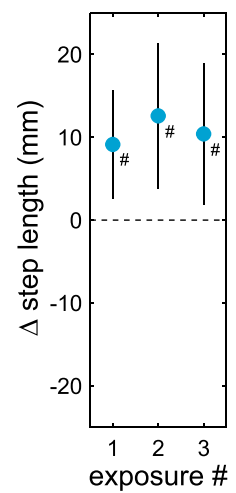

(j)

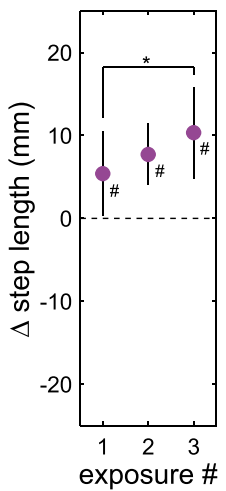

Figure 7. Gait behavior across the three washout periods. The top row (a-e) again illustrates gait metrics during the three washout periods following force-field assistance, while the bottom row $(\mathbf{f}-\mathbf{j})$ illustrates these metrics during washout periods following perturbations. The structure of the figure follows Fig. 5, with $\rho_{\text {disp }}$ representing the partial correlation between pelvis displacement and step width. For these comparisons, the first 225 steps of each exposure are included.

suggesting that the adjustment of active control may require an extensive period of time ${ }^{29}$. Speculatively, we attribute the gradual reduction in the effects of force-field perturbations over repeated exposures to adjustments in the active control used to resist these perturbations, which may otherwise increase the risk of a lateral loss of balance. Changes in $\rho_{\text {disp }}$ during the initial washout period following perturbations provide further evidence for altered active control, with such after-effects previously observed with other perturbing force-fields ${ }^{22,28}$, and commonly cited as a strong indicator of changes in sensorimotor control ${ }^{19}$. The relationship between pelvis displacement and step width was strengthened early in the first washout period but returned to its baseline level-consistent with a return to the initial pattern of active control. However, the changes in $\rho_{\text {disp }}$ during washout periods following repeated perturbation exposures contradicted our hypothesis. Instead of observing decreased after-effects with repeated exposure, step start $\rho_{\text {disp }}$ and step end $\rho_{\text {disp }}$ actually increased significantly. This unexpected result is perhaps due to the fact that the altered active control during these washout periods produces a stronger link between pelvis motion and step width, and thus is unlikely to have a negative effect on balance that would drive further adjustments in active control.

As detailed above, the present results provide evidence for altered sensorimotor control in response to perturbations. Given the extensive prior results demonstrating that humans exert within-step active control over the frontal plane motion of the swing $\operatorname{leg}^{8-14}$, modulation of this control is the most likely explanation for the present results. However, an alternative possibility is that the altered active control takes the form of simply changing the average step characteristics (e.g. step width), which in turn alters the contribution of passive inverted pendular mechanics to step width by changing the initial conditions of the stance leg and trunk at the start of each step. This possibility seems unlikely, as large changes in mean step width and step length from early to late in the Transparent trial were not accompanied by changes in $\rho_{\text {disp }}$. Nevertheless, to gain further insight into the distinction between the expected changes in within-step control of swing leg position and the possible changes in stance leg contributions to step width, we performed a post-hoc analysis. The swing leg contribution to step width was calculated as the mediolateral foot placement - the displacement between the sacrum and the swing heel at the end of the step, as we would expect fluctuations in this measure to be dominated by the frontal plane position of the swing leg. The stance leg contribution to step width was calculated as the mediolateral displacement between the sacrum and the stance heel at the end of the step, which could be highly influenced by passive inverted pendular dynamics of the stance leg and trunk (as well as active control of stance leg musculature). We focused on comparisons across the three force-field exposures, for which our primary analyses revealed the clearest evidence 
(a)

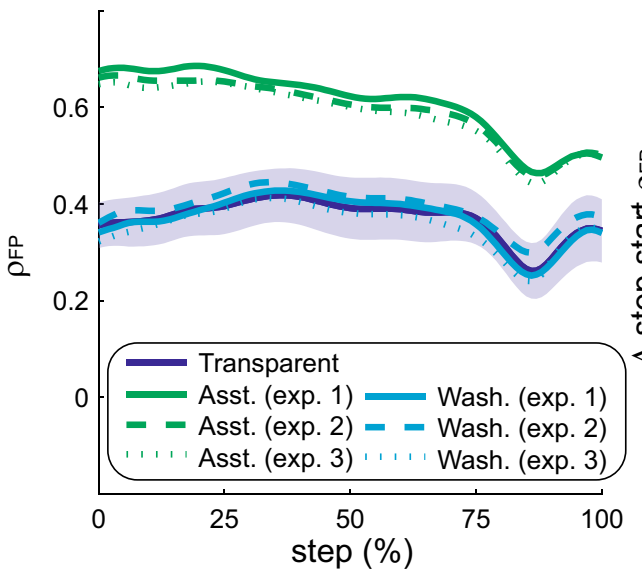

(d)

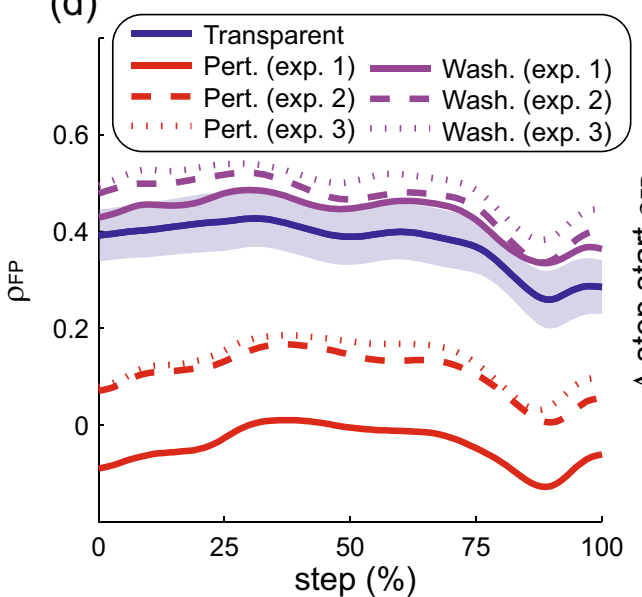

(b)

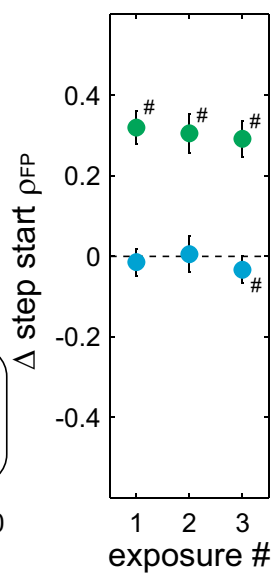

(e)

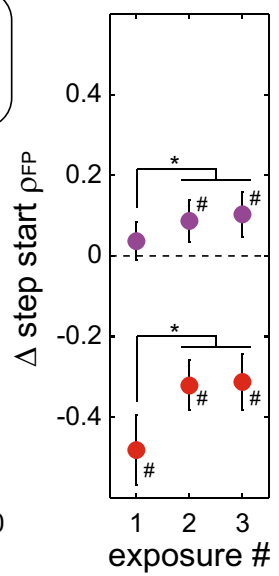

(c)

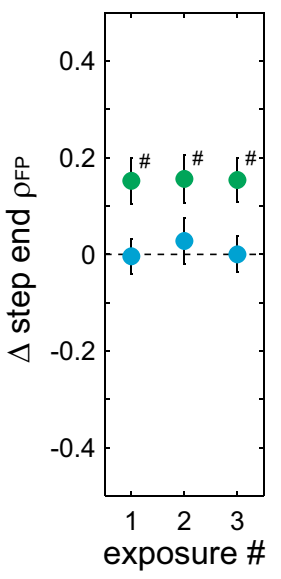

(f)

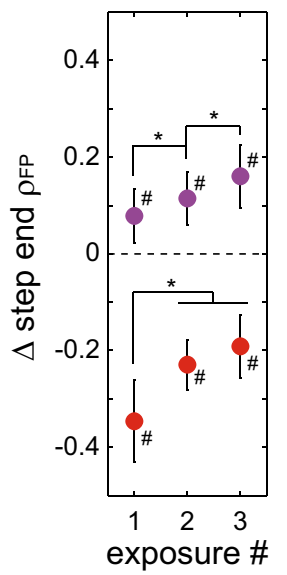

Figure 8. Effects of repeated force-field exposures and washout periods on the partial correlation between pelvis displacement and mediolateral foot placement $\left(\rho_{\mathrm{FP}}\right)$. The top row $(\mathbf{a}-\mathbf{c})$ illustrates gait metrics during and following force-field assistance, while the bottom row (d-f) illustrates these metrics during and following perturbations. The general structure of the figure follows Figs. 6-7. For all comparisons, the first 225 steps of each exposure are included. Asterisks $\left(^{*}\right)$ indicate significant differences between the indicated exposures. Pound signs (\#) indicate a significant difference from the initial Transparent trial.

for perturbation-evoked adaptation. To quantify changes in swing leg control, we modified our primary analysis to calculate the partial correlation between pelvis motion and mediolateral foot placement $\left(\rho_{\mathrm{FP}}\right)$. This metric is illustrated throughout a step for both the direct effects and after-effects of assistance (Fig. 8a) and perturbations (Fig. 8d). The pattern of changes in this swing leg metric is qualitatively near identical to that seen with our primary step width metric $\left(\rho_{\text {disp }}\right)$. Specifically, we observed no change across exposures in the direct effects of assistance on step start $\rho_{\mathrm{FP}}\left(p=0.07\right.$; Fig. 8b) or step end $\rho_{\mathrm{FP}}(p=0.58$; Fig. 8c), or in after-effects of assistance on step start $\rho_{\mathrm{FP}}(p=0.75$; Fig. $8 \mathrm{~b})$ or step end $\rho_{\mathrm{FP}}(p=0.58$; Fig. $8 \mathrm{c})$. Conversely, the effects of perturbations clearly varied across exposures, as the negative direct effects decreased toward baseline in later exposures for both step start $\rho_{\mathrm{FP}}\left(p<0.001\right.$; Fig. 8e) and step end $\rho_{\mathrm{FP}}(p=0.002$; Fig. 8f). As further evidence for altered sensorimotor control of the swing leg, the positive after-effects of perturbations significantly increased with later exposures for both step start $\rho_{\mathrm{FP}}\left(p<0.001\right.$; Fig. 8e) and step end $\rho_{\mathrm{FP}}(p<0.001$; Fig. $8 \mathrm{f})$. To quantify changes that could instead be attributed to altered initial conditions for the stance leg, we calculated the partial correlation between pelvis motion throughout a step and the mediolateral displacement of the sacrum at the end of the step $\left(\rho_{\text {sacrum }}\right)$. This metric is again illustrated throughout a step for both the direct effects and after-effects of assistance (Fig. 9a) and perturbations (Fig. 9c). As this measure trivially converges to one at the end of the step, our statistical analyses focused solely on its value at the start of the step. Unlike our measure of swing leg control, this stance leg metric exhibited no significant changes across three exposures in terms of direct effects of either assistance $(p=0.85$; Fig. 9b) or perturbations ( $p=0.13$; Fig. 9 d), or after-effects of either assistance $(p=0.45$; Fig. 9b) or perturbations ( $p=0.51$; Fig. $9 d$ ). Counter-intuitively, both assistance and perturbations directly increased the strength of the relationship between pelvis displacement early in a step and pelvis displacement at the end of the step. As the force-field did not directly exert forces on the pelvis, perhaps this effect is a generalized response to an 
(a)

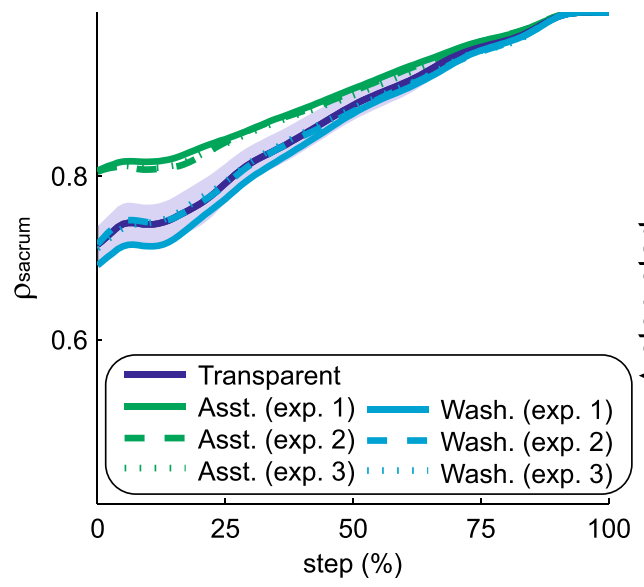

(c)

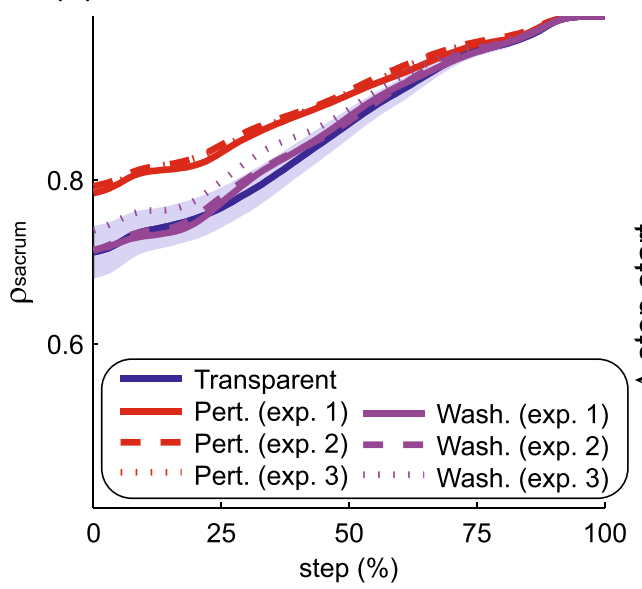

(b)

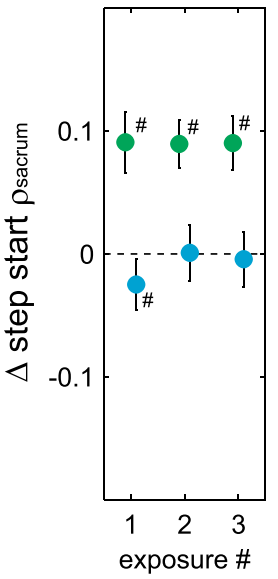

(d)

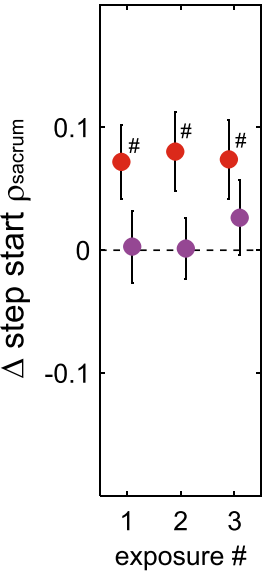

Figure 9. Effects of repeated force-field exposures and washout periods on the partial correlation between mediolateral pelvis displacement throughout a step and mediolateral pelvis displacement at the end of the step $\left(\rho_{\text {sacrum }}\right)$. The top row $(\mathbf{a}-\mathbf{b})$ illustrates gait metrics during and following force-field assistance, while the bottom row $(\mathbf{c}-\mathbf{d})$ illustrates these metrics during and following perturbations. The general structure of the figure follows Fig. 8. For all comparisons, the first 225 steps of each exposure are included.

altered mechanical environment, although further investigation would be needed to address this possibility. These post hoc analyses provide support for altered within-step active control of the swing leg being the most likely explanation for the changes in step width evoked by perturbation exposure.

Although step width and step length often varied both within and across walking trials, we are unable to attribute these changes to the novel mechanical environments produced by the force-field. In general, step width decreased and step length increased over time, changes observed even in the first Transparent trial before assistive or perturbing forces were applied. We suspect that these general trends are not due to the force-field itself, but instead reflect a gradual shift away from a 'cautious' gait pattern with short, wide steps ${ }^{30-33}$ as participants became accustomed to walking while interfaced with the force-field. As most easily seen in Fig. 2, the clearest difference between the effects of the Assistive and Perturbing modes was the wider and shorter steps used while perturbations were delivered. This behavior is likely an example of a 'generalized anticipatory strategy' used by participants to maintain their balance ${ }^{34}$ in response to a context perceived as potentially destabilizing.

While the present results provide evidence for altered active control of step width when perturbed, a limitation of this work is our inability to identify the underlying physiological mechanism. One possibility is errorbased sensorimotor adaptation, in which movement patterns gradually change in response to new mechanical demand $s^{35}$. This adaptation is thought to be driven by sensory prediction errors ${ }^{36}$, as humans seek to reduce the difference between the predicted and sensed movement through a trial-and-error process. In the present work, force-field perturbations could conceivably increase the errors between the intended and actual step width, causing participants to adjust the active muscle contractions typically used to influence swing leg motion ${ }^{8}$. This altered active control could reduce the observed effects of perturbations, as well as produce the tighter link between pelvis motion and step width once the perturbations cease. Unfortunately, calculating our primary outcome measure $\left(\rho_{\text {disp }}\right)$ requires numerous consecutive steps ${ }^{4}$, which prevents us from mimicking analytical approaches 
from split-belt walking that quantify error-based adaptation using a small number of steps (or even single steps). For example, the largest magnitude after-effects are observed for approximately the first five strides following a change in mechanical environment ${ }^{37}$, with the magnitude of such effects influenced by the time course $\mathrm{e}^{24}$, amplitude $^{38}$, and pattern ${ }^{39}$ of the perturbations. Additionally, the time course of adaptation is often calculated based on changes in single step characteristics ${ }^{23}$. Beyond these limitations of our analyses, our observation of increased after-effects with repeated perturbation exposure would not be predicted from error-based adaptation, in which repeated exposure to a novel environment is expected to result in smaller direct and after-effects ${ }^{23-25}$. Perhaps this altered gait behavior would be better explained by the framework of reinforcement learning, in which humans learn new movement patterns based on their perceived value ${ }^{19}$. The tighter link between pelvis displacement and step width may be perceived as valuable for meeting the dual goals of achieving a sufficiently wide step to avoid a lateral loss of balance during the next stance phase $e^{1,2}$ and simultaneously avoiding excessively wide steps that cause larger losses of mechanical energy and increased metabolic cost ${ }^{40}$. The possible distinction between error-based adaptation and reinforcement learning is currently speculative, and carefully designed future work will be required to differentiate between these mechanisms. Additionally, our finding that perturbations influence the active control of step width may be specific to perturbations that target the legs, as recent work has reported that mediolateral trunk perturbations have a more notable effect on control of the trunk ${ }^{41}$.

A further limitation of the present work was the sample size. Participant recruitment was based on our previous study ${ }^{16}$, in which this sample size ( $\mathrm{n}=12$ per group) was sufficient to detect significant direct effects and after-effects of force-field assistance and perturbations. However, the present study's statistical comparisons of changes in $\rho_{\text {disp }}$ within a given mechanical context resulted in numerous p-values between 0.01 and 0.10 , which should be interpreted with caution. Just as the present results supported the primary findings of our original work, future work should attempt to replicate the present results with a larger sample size, based on effect size estimates we can now calculate.

The present results motivate future experiments to provide further insight into the control of step width during walking. For example, the force-field could be applied in an asymmetric fashion, assisting a dynamicallyappropriate step width on one side, while perturbing it on the other. Such work could reveal whether humans are able to differentially modulate the active control of step width in response to simultaneously experienced novel mechanical environments, and may have implications for the asymmetries in this control observed in clinical populations ${ }^{42,43}$. Additionally, future work will expose individual participants to both assistance and perturbations in separate trials, allowing direct comparisons of these approaches and an investigation of potential ordering effects (e.g. whether being assisted after being perturbed has different effects than being perturbed after being assisted). Finally, the present results may have implications for the control of exoskeletons used to assist walking. Several recent devices have integrated adjustments of step width based on the mechanical state of the pelvis or torso ${ }^{44,45}$, generally corresponding to our Assistive approach. Our present results suggest that while this assistance may indeed have the direct effect of improving walking balance, it may be unlikely to encourage users to strengthen their active control of step width - as would be desired with a device for training walking balance.

In conclusion, this study provides initial evidence for altered within-step active control of step width in response to targeted force-field perturbations. With extended periods of exposure to these perturbations, participants exhibited an increased ability to both resist the direct effects of the perturbations and maintain the strengthened link between pelvis motion and step width once the perturbations ceased. The apparent effects of force-field assistance on active control were more modest; participants generally continued to "accept" the provided assistance with repeated exposure, but did not exhibit positive after-effects once the assistance ceased. Future work is needed to investigate the potential motor adaptation or learning mechanisms underlying these results.

\section{Methods}

Participants. This experiment involved 24 young, neurologically-intact participants who had not previously interacted with the force-field. Participants were randomly assigned to either the Assistive group ( $\mathrm{n}=12$; age $=22 \pm 1$ years; height $=170 \pm 9 \mathrm{~cm}$; mass $=70 \pm 13 \mathrm{~kg}$; mean $\pm \mathrm{SD})$ or the Perturbing group $(\mathrm{n}=12$; age $=23 \pm 1$ years; height $=170 \pm 8 \mathrm{~cm}$; mass $=65 \pm 13 \mathrm{~kg}$; mean $\pm S D$ ). This study was approved by the Medical University of South Carolina Institutional Review Board, all methods were performed in accordance with the approved guidelines, and all participants provided written informed consent.

Force-field design and control. We used a custom-designed force-field to exert mediolateral forces on participants' legs while walking. This force-field has previously been described in detail ${ }^{15}$ and used to both assist and perturb the relationship between mediolateral pelvis motion and step width ${ }^{16}$. Pairs of linear actuators (UltraMotion; Cutchoge, NY, USA) positioned anterior and posterior to a treadmill were used to rapidly adjust the mediolateral location of two steel wires running parallel to the treadmill belts, and in series with extension springs. These wires passed through leg cuffs worn on the lateral shank, which allowed free anteroposterior and vertical leg motion. Participants experienced mediolateral leg forces that were proportional to the mediolateral displacement between the actuator end point and the leg cuff ${ }^{15}$. The force-field's mediolateral stiffness (ratio between mediolateral force and displacement) was $180 \mathrm{~N} / \mathrm{m}$, based on the results of a pilot study in which this stiffness was sufficient to produce clear effects on step width (Appendix A).

Three force-field control modes were applied: Transparent, Assistive, and Perturbing. For all modes, actuator positions were controlled based on the location of active LED markers (PhaseSpace; San Leandro, CA, USA) on the sacrum, heels, and/or leg cuffs. The sacrum marker was used to estimate mediolateral pelvis location, a simplification previously found to have minimal effects on our calculations ${ }^{4}$. In Transparent mode, each actuator followed the mediolateral motion of the corresponding leg cuff, minimizing the resultant mediolateral leg forces. 
In both the Assistive and Perturbing modes, we first used the mediolateral displacement of the pelvis from the stance heel at the start of each step to predict a dynamically-appropriate step width. For example, if a right step began with the pelvis located relatively far to the right of the left stance heel, we would predict that the upcoming step should be relatively wide. In the Assistive mode, we positioned the actuators to push the swing leg toward the predicted dynamically-appropriate step width. This was done using the following equation, in which $S W$ represents the predicted step width, $x_{\text {pelvis }}$ represents the mediolateral displacement of the pelvis from the stance heel at the start of the step, and $S W_{\text {mean }}$ represents the participant's mean step width:

$$
S W=1.12 * x_{\text {pelvis }}+0.47 * S W_{\text {mean }}
$$

This equation was directly derived from previously collected empirical data quantifying the relationship between pelvis displacement and step width among young, neurologically-intact control participants ${ }^{4}$. The positive coefficient of $x_{\text {pelvis }}$ indicates that for larger mediolateral pelvis displacements, the force-field pushes the swing leg toward a wider step width. The inclusion of the $S W_{\text {mean }}$ term accounts for a participant's average step width, which varies across individuals. The coefficient for this term was also derived from control behavior, as we found the average step width to typically be preceded by a mediolateral pelvis displacement of $0.47^{\star} S W_{\text {mean }}$ at the start of the step. From this equation, a pelvis displacement of $0.47^{\star} S W_{\text {mean }}$ would encourage a step width of $S W_{\text {mean }}$.

Conversely, in the Perturbing mode, we positioned the actuators to push the swing leg away from the predicted step width (e.g. to encourage a narrow step when a wide step would be dynamically appropriate). This was accomplished using the following equation:

$$
\mathrm{SW}=-1.12 * x_{\text {pelvis }}+1.53 * S W_{\text {mean }}
$$

In this equation, the negative coefficient of $x_{\text {pelvis }}$ indicates that for larger mediolateral pelvis displacements, the force-field pushes the swing leg toward a narrower step width. We again accounted for a participant's average step width by including a $S W_{\text {mean }}$ term. The coefficient for this term was chosen such that the typical mediolateral pelvis displacement of $0.47^{\star} S W_{\text {mean }}$ would again encourage a step width of $S W_{\text {mean }}$, just as with the Assistive equation. Therefore, the structure of the Assistive and Perturbing equations does not inherently push participants to walk with wider or narrower average steps.

The effects of these force-field control equations were first investigated in our prior work ${ }^{16}$, along with several other equations of varying complexity. While all previously tested control equations had similar effects, we observed the largest positive after-effects following force-field perturbations with the equation applied here ${ }^{16}$.

With the applied control, the mediolateral forces acting on participants' legs are not directly controlled, and are not constant for each step. Instead, the force-field wires simply move to the prescribed mediolateral location, and the force experienced by users is proportional to their leg's mediolateral deviation from this location. So aligning the legs with the wires would result in low experienced forces, while positioning the leg far from the wires would result in relatively high forces.

Experimental procedure. Participants performed five treadmill walking trials at $1.2 \mathrm{~m} / \mathrm{s}$, in non-randomized order. All participants wore a harness attached to an overhead rail that did not support body weight but would have prevented a fall in case of a loss of balance. Participants first walked for 5-min without interacting with the force-field (Normal) as a familiarization period, followed by a 5 -min trial with the force-field in Transparent mode. Data from the final 2-min of the Normal trial were used to calculate each participant's mean step width, with this value integrated into the relevant Assistive or Perturbing control equation. Participants in the Assistive group then performed three identical 10-min trials in which the force-field was in Assistive mode for the first 5-min and Transparent mode for the final 5-min as a washout period. Participants in the Perturbing group performed three corresponding 10-min trials in which the force-field was in Perturbing mode for the first 5-min and Transparent mode for the final 5-min, again as a washout period.

Data collection and processing. Active LED marker locations were sampled at $120 \mathrm{~Hz}$ and low-pass filtered at $10 \mathrm{~Hz}$. We defined each step start as the time point when the ipsilateral heel velocity changed from posterior to anterior ${ }^{46}$. The step end was defined as the time point when the contralateral heel velocity changed from posterior to anterior. Throughout each step, we quantified the mediolateral displacement of the pelvis relative to the stance heel, as well as the mediolateral pelvis velocity. Step width was defined as the mediolateral displacement between the ipsilateral heel marker at the step end and the contralateral heel marker at the step start. Step length was calculated as the difference between the anterior position of the ipsilateral heel at the step end and the anterior position of the contralateral heel at the previous step end, accounting for treadmill speed.

Each 5-min walking period within a given force-field mode was divided into five "bins" for the steps taken with each leg: steps 1-45; steps 46-90; steps 91-135; steps 136-180; steps 181-225. While the number of steps taken within each 5 -min period varied fairly widely across participants and conditions $(275 \pm 12$; mean \pm s.d. $)$, every participant took at least 225 steps with each leg for each 5 -min period. Comparisons across bins thus included the same number of steps (interactions with the novel mechanical environment), while the inclusion of 45 consecutive steps allowed convergence of our correlation-based measures (detailed below) ${ }^{4}$. An alternative approach of dividing walking trials into bins based on time (i.e. minutes 1-5) produced similar results (Appendix B).

Our primary outcome measure was the partial correlation between mediolateral pelvis displacement and step width $\left(\rho_{\text {disp }}\right)$, accounting for variation in mediolateral pelvis velocity ${ }^{16}$. The strength of the relationship between pelvis motion and step width can alternatively be quantified using the $\mathrm{R}^{2}$ magnitude calculated by performing a linear regression between these gait variables ${ }^{4}$. Such $\mathrm{R}^{2}$-based analyses essentially parallel the changes in $\rho_{\text {disp }}$ 
(Appendix C), likely because predictions of step width seem to be dominated by pelvis displacement during steady-state walking ${ }^{4}$.

For illustrative purposes, we calculated $\rho_{\text {disp }}$ over the course of a step for each 45 -step bin. We resampled pelvis displacement and velocity values during each step to create 101-sample vectors, thus allowing comparisons across steps of variable periods. We created a trajectory of $\rho_{\text {disp }}$ values by calculating $\rho_{\text {disp }}$ from the pelvis displacement and velocity values at each normalized time point in the step (from 0-100) and the step width values at the end of the step. While we calculated $\rho_{\text {disp }}$ throughout the step, our statistical analyses focused on $\rho_{\text {disp }}$ at the start and end of the step, as well as step width and step length.

Statistics. Upon visual inspection, the gait metrics of interest appeared consistent with a normal distribution. However, we chose to apply non-parametric statistical methods due to the relatively small sample size in this study ${ }^{47}$. We first performed a series of Friedman's tests $(\alpha=0.05)$ to compare three time periods: late in the initial Normal trial (steps 181-225, a period by which we expect participants to be familiarized with treadmill walking), early in the Transparent trial (steps 1-45), and late in the Transparent trial (steps 181-225). These comparisons included all 24 participants, and compared each of our gait metrics (step start $\rho_{\text {disp, }}$, step end $\rho_{\text {disp, }}$, step width, step length). Our subsequent statistical tests were focused on specific hypotheses, and were performed separately for participants in the Assistive and Perturbing groups. To test whether the direct effects of the initial force-field exposure changed over time, we performed Wilcoxon signed-rank tests $(\alpha=0.05)$ to compare each of our gait metrics between early (steps 1-45) and late (steps 181-225) in the first exposure to the Assistive/ Perturbing force-field. To test whether potential after-effects that follow the initial force-field exposure changed over time, we performed identically structured Wilcoxon signed-rank tests $(\alpha=0.05)$ to compare these gait metrics between early (steps 1-45) and late (steps 181-225) in the Transparent washout period that followed the first exposure to Assistance/Perturbations. To test whether repeated exposure to the altered mechanical environment influenced the direct effects of the force-field, we performed Friedman's tests $(\alpha=0.05)$ to compare these gait metrics (combined across all 5 bins) between the three exposures to the Assistive/Perturbing force-field. Finally, to test whether repeated exposure to the altered mechanical environment influenced the magnitude of any aftereffects, we performed identical Friedman's tests $(\alpha=0.05)$ to compare these gait metrics (again, combined across all 5 bins) between the three washout periods in Transparent mode. In the case of a significant effect for a Friedman's test, we performed Wilcoxon signed-rank tests ( $\alpha=0.017$ to account for multiple comparisons) to detect significant differences between individual conditions.

\section{Data availability}

The datasets generated during the current study are available from the corresponding author on reasonable request.

Received: 31 December 2019; Accepted: 2 July 2020

Published online: 22 July 2020

\section{References}

1. MacKinnon, C. D. \& Winter, D. A. Control of whole body balance in the frontal plane during human walking. J. Biomech. 26, 633-644 (1993).

2. Bruijn, S. M. \& van Dieen, J. H. Control of human gait stability through foot placement. J. R. Soc. Interface 15, 20170816 (2018).

3. Wang, Y. \& Srinivasan, M. Stepping in the direction of the fall: the next foot placement can be predicted from current upper body state in steady-state walking. Biol. Lett. 10, 20140405 (2014).

4. Stimpson, K. H., Heitkamp, L. N., Horne, J. S. \& Dean, J. C. Effects of walking speed on the step-by-step control of step width. J. Biomech. 68, 78-83 (2018).

5. Patil, N. S., Dingwell, J. B. \& Cusumano, J. P. Correlations of pelvis state to foot placement do not imply within-step active control. J. Biomech. 97, 109375 (2019).

6. Kuo, A. D. Stabilization of lateral motion in passive dynamic walking. Int. J. Rob. Res. 18, 917-930 (1999).

7. Joshi, V. \& Srinivasan, M. A controller for walking derived from how humans recover from perturbations. J. R. Soc. Interface 16, 20190027 (2019).

8. Rankin, B. L., Buffo, S. K. \& Dean, J. C. A neuromechanical strategy for mediolateral foot placement in walking humans. J. Neurophysiol. 112, 374-383 (2014).

9. Hof, A. L. \& Duysens, J. Response of human hip abductor muscles to lateral balance perturbations during walking. Exp. Brain. Res. 230, 301-310 (2013).

10. Afschrift, M. et al. Modulation of gluteus medius activity reflects the potential of the muscle to meet the mechanical demands during perturbed walking. Sci. Rep. 8, 11675 (2018).

11. Roden-Reynolds, D. C., Walker, M. H., Wasserman, C. R. \& Dean, J. C. Hip proprioceptive feedback influences the control of mediolateral stability during human walking. J. Neurophysiol. 114, 2220-2229 (2015).

12. Arvin, M. et al. Where to step? Contributions of stance leg muscle spindle afference to planning of mediolateral foot placement for balance control in young and old adults. Front. Physiol. 9, 1134 (2018).

13. Stokes, H. E., Thompson, J. D. \& Franz, J. R. The neuromuscular origins of kinematic variability during perturbed walking. Sci. Rep. 7, 808 (2017).

14. Reimann, H., Fettrow, T., Thompson, E. D. \& Jeka, J. J. Neural control of balance during walking. Front. Physiol. 9, 1271 (2018).

15. Nyberg, E. T., Broadway, J., Finetto, C. \& Dean, J. C. A novel elastic force-field to influence mediolateral foot placement during walking. IEEE Trans. Neur. Syst. Rehabil. Eng. 25, 1481-1488 (2017).

16. Heitkamp, L. N., Stimpson, K. H. \& Dean, J. C. Application of a novel force-field to manipulate the relationship between pelvis motion and step width in human walking. IEEE. Trans. Neur. Syst. Rehabil. Eng. 27, 2051-2058 (2019).

17. Reisman, D. S., Block, H. J. \& Bastian, A. J. Interlimb coordination during locomotion: what can be adapted and stored?. J. Neurophysiol. 94, 2403-2415 (2005).

18. Reisman, D. S., Bastian, A. J. \& Morton, S. M. Neurophysiologic and rehabilitation insights from the split-belt and other locomotor adaptation paradigms. Phys. Ther. 90, 187-195 (2010). 
19. Roemmich, R. T. \& Bastian, A. J. Closing the loop: from motor neuroscience to neurorehabilitation. Ann. Rev. Neurosci. 41, 415-429 (2018).

20. Yen, S. C., Schmit, B. D., Landry, J. M., Roth, H. \& Wu, M. Locomotor adaptation to resistance during treadmill training transfers to overground walking in human SCI. Exp. Brain. Res. 216, 473-482 (2012).

21. Kim, S. H. et al. Robot-assisted modifications of gait in healthy individuals. Exp. Brain. Res. 202, 809-824 (2010).

22. Kao, P. C., Srivastava, S., Agrawal, S. K. \& Scholz, J. P. Effect of robotic performance-based error-augmentation versus errorreduction training on the gait of healthy individuals. Gait Posture 37, 113-120 (2013).

23. Malone, L. A., Vasudevan, E. V. L. \& Bastian, A. J. Motor adaptation training for faster relearning. J. Neurosci. 31, 15136-15143 (2011).

24. Day, K. A., Leech, K. A., Roemmich, R. T. \& Bastian, A. J. Accelerating locomotor saving in learning: compressing four training days to one. J. Neurophysiol. 119, 2100-2113 (2018).

25. Leech, K. A., Day, K. A., Roemmich, R. T. \& Bastian, A. J. Movement and perception recalibrate differently across multiple days of locomotor learning. J. Neurophysiol. 120, 2130-2137 (2018).

26. Reinkensmeyer, D.J., Akoner, O.J., Ferris, D.P, Gordon, K.E. Slacking by the human motor system: computational models and implications for robotic orthoses. In Conference Proceedings IEEE Engineering in Medicine and Biology Society 2009, 2129-2132 (2009).

27. Cajigas, I., Koenig, A., Severini, G., Smith, M. \& Bonato, P. Robot-induced perturbations of human walking reveal a selective generation of motor adaptation. Sci. Robot. 2, eaam7749 (2017).

28. Cajigas, I., Goldsmith, M. T., Duschau-Wicke, A., Riener, R., Smith, M. A., Brown, E. N., Bonato, P. Assessment of lower extremity motor adaptation via an extension of the force field adaptation paradigm. In 2010 Conference Proceedings IEEE Engineering Medicine and Biology Society, pp. 4522-4525 (2010).

29. Sanchez, N., Simha, S. N., Donelan, J. M. \& Finley, J. M. Taking advantage of external mechanical work to reduce metabolic cost: the mechanics and energetics of split-belt treadmill walking. J. Physiol. 597, 4053-4068 (2019).

30. Chamberlin, M. E., Fulwider, B. D., Sanders, S. L. \& Medeiros, J. M. Does fear of falling influence spatial and temporal gait parameters in elderly persons beyond changes associated with normal aging?. J. Gerontol. Med. Sci. 60A, 1163-1167 (2005).

31. Hak, L. et al. Speeding up or slowing down? Gait adaptations to preserve gait stability in response to balance perturbations. Gait Posture 36, 260-264 (2012).

32. Monsch, E. D., Franz, C. O. \& Dean, J. C. The effects of gait strategy on metabolic rate and indicators of stability during downhill walking. J. Biomech. 45, 1928-1933 (2012).

33. Hubbuch, J. E., Bennett, B. W. \& Dean, J. C. Proprioceptive feedback contributes to the adaptation toward an economical gait pattern. J. Biomech. 48, 2925-2931 (2015).

34. Wu, M., Matsubara, J. H. \& Gordon, K. E. General and specific strategies used to facilitate locomotor maneuvers. PLoS ONE 10, e0132707 (2015).

35. Bastian, A. J. Understanding sensorimotor adaptation and learning for rehabilitation. Curr. Opin. Neurol. 21, 628-633 (2008).

36. Tseng, Y., Diedrichsen, J., Krakauer, J. W., Shadmehr, R. \& Bastian, A. J. Sensory prediction errors drive cerebellum-dependent adaptation of reaching. J. Neurophysiol. 98, 54-62 (2007).

37. Malone, L. A. \& Bastian, A. J. Thinking about walking: effects of conscious correction versus distraction on locomotor adaptation. J. Neurophysiol. 103, 1954-1962 (2010).

38. Leech, K. A., Roemmich, R. T. \& Bastian, A. J. Creating flexible motor memories in human walking. Sci. Rep. 8, 94 (2018).

39. Torres-Oveido, G. \& Bastian, A. J. Natural error patterns enable transfer of motor learning to novel contexts. J. Neurophysiol. 107, 346-356 (2012).

40. Donelan, J. M., Kram, R. \& Kuo, A. D. Mechanical and metabolic determinants of the preferred step width in human walking. Proc. Biol. Sci. 268, 1985-1992 (2001).

41. Best, A. N., Martin, J. M., Li, Q. \& Wu, A. R. Stepping behaviour contributes little to balance control against continuous mediolateral trunk perturbations. J. Exp. Biol. 222, 212787 (2019).

42. Stimpson, K. H., Heitkamp, L. N., Embry, A. E. \& Dean, J. C. Post-stroke deficits in the step-by-step control of paretic step width. Gait Posture 70, 136-140 (2019).

43. Dean, J. C. \& Kautz, S. A. Foot placement control and gait instability among people with stroke. J. Rehabil. Res. Dev. 52, 577-590 (2015).

44. Wang, S. et al. Design and control of the MINDWALKER exoskeleton. IEEE Trans. Neur. Syst. Rehabil. 23, 277-286 (2014).

45. Zhang, T., Tran, M. \& Huang, H. Design and experimental validation of hip exoskeleton with balance capacities for walking assistance. IEEE Trans. Mechantron. 23, 274-285 (2018).

46. Zeni, J. A., Richards, J. G. \& Higginson, J. S. Two simple methods for determining gait events during treadmill and overground walking using kinematic data. Gait Posture 27, 710-714 (2008).

47. Morgan, C. J. Use of proper statistical techniques for research studies with small samples. Am. J. Physiol. Lung Cell Mol. Physiol. 313, L873-L877 (2017).

\section{Acknowledgements}

This work was supported by a grant from the National Science Foundation (1603391).

\section{Author contributions}

JD conceived of the research. NR created software used in data collections. AC, RH, HK, NR, and LW conducted data collections. NR, LW, and JD contributed to data analysis. JD drafted the initial manuscript. All authors reviewed and revised the manuscript.

\section{Competing interests}

The authors declare no competing interests.

\section{Additional information}

Supplementary information is available for this paper at https://doi.org/10.1038/s41598-020-69052-5.

Correspondence and requests for materials should be addressed to J.C.D.

Reprints and permissions information is available at www.nature.com/reprints.

Publisher's note Springer Nature remains neutral with regard to jurisdictional claims in published maps and institutional affiliations. 
(c) (i) Open Access This article is licensed under a Creative Commons Attribution 4.0 International cc) License, which permits use, sharing, adaptation, distribution and reproduction in any medium or format, as long as you give appropriate credit to the original author(s) and the source, provide a link to the Creative Commons license, and indicate if changes were made. The images or other third party material in this article are included in the article's Creative Commons license, unless indicated otherwise in a credit line to the material. If material is not included in the article's Creative Commons license and your intended use is not permitted by statutory regulation or exceeds the permitted use, you will need to obtain permission directly from the copyright holder. To view a copy of this license, visit http://creativecommons.org/licenses/by/4.0/.

This is a U.S. Government work and not under copyright protection in the US; foreign copyright protection may apply 2020 